\title{
Genetic evidence implicating DARPP-32 in human frontostriatal structure, function, and cognition
}

\author{
Andreas Meyer-Lindenberg, ${ }^{1,2,3}$ Richard E. Straub, ${ }^{3}$ Barbara K. Lipska, ${ }^{3}$ Beth A. Verchinski, ${ }^{2,3}$ \\ Terry Goldberg, ${ }^{3}$ Joseph H. Callicott, ${ }^{3}$ Michael F. Egan,, ${ }^{3}$ Stephen S. Huffaker, ${ }^{3}$ \\ Venkata S. Mattay, ${ }^{2,3}$ Bhaskar Kolachana, ${ }^{3}$ Joel E. Kleinman, ${ }^{3}$ and Daniel R. Weinberger ${ }^{3}$ \\ ${ }^{1}$ Unit for Systems Neuroscience in Psychiatry, ${ }^{2}$ Neuroimaging Core Facility, and ${ }^{3}$ Clinical Brain Disorders Branch, \\ Genes, Cognition, and Psychosis Program, National Institute for Mental Health (NIMH), NIH, \\ US Department of Health and Human Services, Bethesda, Maryland, USA.
}

\begin{abstract}
Dopamine- and cAMP-regulated phosphoprotein of molecular weight $32 \mathrm{kDa}$ (DARPP-32), encoded by $P P P 1 R 1 B$, is a pivotal integrator of information in dopaminoceptive neurons, regulating the response to neuroleptics, psychotomimetics, and drugs of abuse, and affecting striatal function and plasticity. Despite extensive preclinical work, there are almost no data on DARPP-32 function in humans. Here, we identify, through resequencing in 298 chromosomes, a frequent PPP1R1B haplotype predicting mRNA expression of PPP1R1B isoforms in postmortem human brain. This haplotype was associated with enhanced performance on several cognitive tests that depend on frontostriatal function. Multimodal imaging of healthy subjects revealed an impact of the haplotype on neostriatal volume, activation, and the functional connectivity of the prefrontal cortex. The haplotype was associated with the risk for schizophrenia in 1 family-based association analysis. Our convergent results identify a prefrontal-neostriatal system affected by variation in PPP1R1B and suggest that DARPP-32 plays a pivotal role in cognitive function and possibly in the pathogenesis of schizophrenia.
\end{abstract}

\section{Introduction}

Dopamine- and cAMP-regulated phosphoprotein of molecular weight $32 \mathrm{kDa}$ (DARPP-32) was initially identified as a major target for dopamine-activated adenylyl cyclase in striatum (1). DARPP-32 acts as an amplifier of PKA- and PKG-mediated signaling when it is phosphorylated at Thr34, which converts it into an inhibitor of a multifunctional serine/threonine protein phosphatase, PP-1 (2). Conversely, phosphorylation at Thr75 by Cdk 5 converts DARPP-32 into an inhibitor of PKA. Over more than 2 decades, an intense research effort has demonstrated that this dual function places DARPP-32 at a unique position as a central molecular switch, integrating multiple information streams and converging through a variety of neurotransmitters, neuromodulators, neuropeptides, and steroid hormones onto dopaminoceptive neurons (3). Since dopaminergic neurotransmission is critical for motivated behavior, working memory (4), and reward-related learning (5) and is implicated in, among other conditions, schizophrenia (6), alcoholism (7), Parkinson disease, and pathological gambling (8), DARPP-32 has received considerable attention not only in basic neuroscience but also in studies of the pathogenesis of these disorders and as a potential drug target. It has been shown that DARPP-32 mediates effects of D2 receptor stimulation (3), functions as a key node in a final common pathway of psychotomimetics in both frontal cortex and striatum (9), and is implicated in the mechanism of action of drugs of abuse (10).

Nonstandard abbreviations used: COMT, catechol-O-methyltransferase; DARPP-32, dopamine- and cAMP-regulated phosphoprotein of molecular weight $32 \mathrm{kDa}$; DLPFC, dorsolateral prefrontal cortex; FBAT, Family-Based Association Testing; fMRI, functional MRI; FMT, face-matching task; LD, linkage disequilibrium; ROI, region(s) of interest; t-DARPP, truncated DARPP; VBM, voxel-based morphology. Conflict of interest: The authors have declared that no conflict of interest exists. Citation for this article: J. Clin. Invest. 117:672-682 (2007). doi:10.1172/JCI30413.
DARPP-32 is expressed in regions receiving dopaminergic innervation. By far the highest levels are found in the neostriatum (caudate and putamen) (11), where DARPP-32 is expressed in GABAergic medium-sized spiny neurons (12). In a topographically well-organized manner, the neostriatum receives excitatory glutamatergic projections from the cortex and thalamus, integrates them with monoaminergic inputs, and sends them via the globus pallidus and substantia nigra pars reticulata to the thalamus, which projects back to the cortex (13). These parallel processing loops are critical for the ongoing processing of sensorimotor, cognitive, and emotional information (13). In addition, a general role in learning through a reciprocal influence on cortical processing via striatopallidal-thalamocortical pathways has been suggested (14). Of particular interest for neuropsychiatry is a circuit linking the dorsolateral prefrontal cortex (DLPFC) with the rostral striatum (including both the caudate nucleus and the putamen rostral to the anterior commissure) (15). One function attributed to these prefrontal-striatal interactions is that of acting as a "filter" of information competing for prefrontal cortical processing (16). Consequently, lesions to the neostriatal-prefrontal system at all levels in animals and in patients with various striatal disorders impair prefrontally dependent cognitive functions such as working memory, set shifting, and executive control (17); these impairments are characteristic of the cognitive deficits found in conditions such as Parkinson disease and schizophrenia (18).

Despite the considerable importance of DARPP-32 for dopaminergic signaling and striatal function in animal models and the potentially substantial clinical implications, data demonstrating the relevance of DARPP-32 in humans are almost wholly lacking. One report found that DARPP-32 protein abundance was selectively reduced in the prefrontal cortex of brain tissue from patients with schizophrenia (19), and 1 recent report found no significant 


\section{Table 1}

Marker and map information in the CBDB/NIMH sample

\begin{tabular}{|c|c|c|c|c|c|c|c|c|c|}
\hline M no. & $\begin{array}{l}\text { dbSNP } \\
\text { rs no. }{ }^{A}\end{array}$ & $\begin{array}{l}\text { Coding } \\
\text { strand SNP }\end{array}$ & $\begin{array}{c}\text { Chromosome } \\
\text { location }^{B}\end{array}$ & $\begin{array}{l}\text { Intermarker } \\
\text { distance }\end{array}$ & $\begin{array}{c}\text { Distance } \\
\text { from marker } 1\end{array}$ & $\begin{array}{l}\text { Cases: } \\
\text { MAFC }\end{array}$ & $\begin{array}{l}\text { Cases: } \\
\text { HWED }\end{array}$ & $\begin{array}{c}\text { Controls: } \\
\text { MAFC }\end{array}$ & $\begin{array}{l}\text { Controls: } \\
\text { HWED }\end{array}$ \\
\hline M01 & Novel & $T / G$ & 35033572 & 0 & 0 & 0.092 & 0.70 & 0.077 & 1.0 \\
\hline M02 & Novel & $\mathrm{T} / \mathrm{A}$ & 35033822 & 250 & 250 & 0.28 & 0.42 & 0.28 & 0.62 \\
\hline M03 & rs4795390 & $\mathrm{C} / \mathrm{G}$ & 35035136 & 1314 & 1564 & 0.15 & 0.20 & 0.16 & 0.62 \\
\hline M04 & rs879606 & $\mathrm{G} / \mathrm{A}$ & 35035375 & 239 & 1803 & 0.14 & 0.16 & 0.17 & 1.0 \\
\hline M05 & Novel & $\mathrm{G} / \mathrm{A}$ & 35036752 & 1377 & 3180 & 0.043 & - & 0.031 & - \\
\hline M06 & Novel & $\mathrm{G} / \mathrm{A}$ & 35038003 & 1251 & 4431 & 0.056 & - & 0.088 & - \\
\hline M07 & Novel & $G / A$ & 35038422 & 419 & 4850 & 0.043 & - & 0.031 & - \\
\hline M08 & rs2271309 & $A / G$ & 35038516 & 94 & 4944 & 0.35 & RFA & 0.40 & RFA \\
\hline M09 & Novel & $T / G$ & 35038726 & 210 & 5154 & 0.077 & - & 0.065 & - \\
\hline M10 & Novel & $\mathrm{A} / \mathrm{G}$ & 35040577 & 1851 & 7005 & 0.077 & - & 0.065 & - \\
\hline M11 & rs11651497 & $\mathrm{C} / \mathrm{T}$ & 35043524 & 2947 & 9952 & 0.21 & 0.22 & 0.23 & 0.71 \\
\hline M12 & rs907094 & $\mathrm{A} / \mathrm{G}$ & 35043897 & 373 & 10325 & 0.21 & 0.32 & 0.23 & 0.57 \\
\hline M13 & rs3764353 & $\mathrm{C} / \mathrm{T}$ & 35044307 & 410 & 10735 & 0.21 & 0.32 & 0.23 & 0.46 \\
\hline M14 & rs3764352 & $\mathrm{T} / \mathrm{C}$ & 35044465 & 158 & 10893 & 0.21 & 0.22 & 0.23 & 0.45 \\
\hline M15 & rs3794712 & $\mathrm{C} / \mathrm{A}$ & 35045013 & 548 & 11441 & 0.17 & 0.087 & 0.18 & 0.26 \\
\hline M16 & Novel & $G / A$ & 35045616 & 603 & 12044 & 0.030 & - & 0.035 & - \\
\hline M17 & rs734645 & $A / G$ & 35045819 & 203 & 12247 & 0.078 & - & 0.062 & - \\
\hline
\end{tabular}

M08 deviated from Hardy-Weinberg equilibrium (HWE) and was not analyzed further. SNPs with minor allele frequency (MAF) of less than 0.1 were not tested for deviation from HWE. ASingle Nucleotide Polymorphism database RefSNP accession ID number (http://www.ncbi.nlm.nih.gov/projects/SNP/); BUCSC March 2006 assembly; ${ }^{C} P$ values; Dexact $P$ values. See Figure 1 for map of gene. RFA, removed from further analyses. Family-based association sample (257 families of white ancestry, CBDB/NIMH sample). See Subjects in Methods for precise breakdown.

difference in mRNA levels in brains from 16 elderly schizophrenic patients compared with matched controls (20). However, given the central role of the protein in molecular mechanisms related to dopaminergic neurotransmission in striatum and its diverse pharmacological actions, we hypothesized that it would be associated in humans with higher-level cognitive and emotional behaviors implicated in cortical-striatal circuitry and function and contribute genetically to variation in these functions. Since genetic variation does not directly cause behavioral phenotypes but rather impacts on neuronal features that influence neural systems-level processing (21), we used a hierarchical stepwise translational genetic approach to investigate this issue. First, we identified genetic variation in PPP1R1B through resequencing. The common variants, only present in noncoding DNA, were then genotyped in a large family-based dataset of white subjects, the Clinical Brain Disorders Branch/NIMH (CBDB/NIMH) sample, identifying genetic variants (SNPs and haplotypes) that were associated with performance on a range of cognitive tests dependent on frontostriatal function. Since this suggested an impact on neuronal function, we studied, in an independent postmortem dataset, the influence of genetic variation in PPP1R1B on PPP1R1B mRNA levels, as transcriptional regulation is a frequently discussed mechanism for the impact of nonexonic genetic variation on neuronal function in humans (22). We found that the same variants that had an impact on cognitive function predicted mRNA expression of PPP1R1B isoforms in postmortem human brain. Together with the cognitive data, this indicated a molecular mechanism for the functional influence of $P P P 1 R 1 B$ genetic variation on striatal neurons and their likely interactions with prefrontal cortex. In an independent and large sample of healthy white subjects, we tested this possibility on the neural systems level using multimodal neuroimaging. We found that these same genetic variants in $P P P 1 R 1 B$ had an impact on neostriatal structure and activation as well as on structural and functional connectivity of striatum with prefrontal cortex. As an active genetic control, we studied the well-characterized Val108/158 Met polymorphism in catechol-O-methyltransferase $(C O M T)$. This variant is useful as a stringent control, as it is associated with prefrontal cognitive function and functional brain activation $(23,24)$ as well as schizophrenia and has been shown to have a strong impact on dopamine turnover, but preferentially in prefrontal cortex (25), not in the striatum (26). Indeed, we found that this polymorphism had no effect on striatal volume or activation or PPP1R1B mRNA expression. Finally, we provide preliminary evidence though analysis of the CBDB/NIMH family-based association dataset that the same variants may also be associated with risk for schizophrenia, a disease in which disturbances of dopaminergic neurotransmission and frontostriatal function play a prominent role $(27,28)$.

\section{Results}

Genetic variation in PPP1R1B. Resequencing of DNA from 105 white and 44 African American probands identified 17 SNPs with minor allele frequencies in the white sample greater than $3 \%$ (Table 1). These were then genotyped in a large family-based sample of white subjects, the CBDB/NIMH family dataset. Overall, linkage disequilibrium (LD) between markers was moderately strong (Figure 1). No coding or splice site SNPs or other obvious common functional variants were identified. Using the method of Gabriel et al. (29), a large haplotype block spanning M02-M15 was identified (Figure 1). From the genotyped variants, to avoid multiple testing, we selected SNPs M03, M04, and M11-M15 within that block as a 7-SNP haplotype characterizing common variations in DARPP-32 for purposes of clinical association, gene expression analyses, and multimodal neuroimaging, all performed in independent datasets of white subjects. These 7 SNPs were selected because they showed the strongest individual SNP associations in the cognitive phe- 


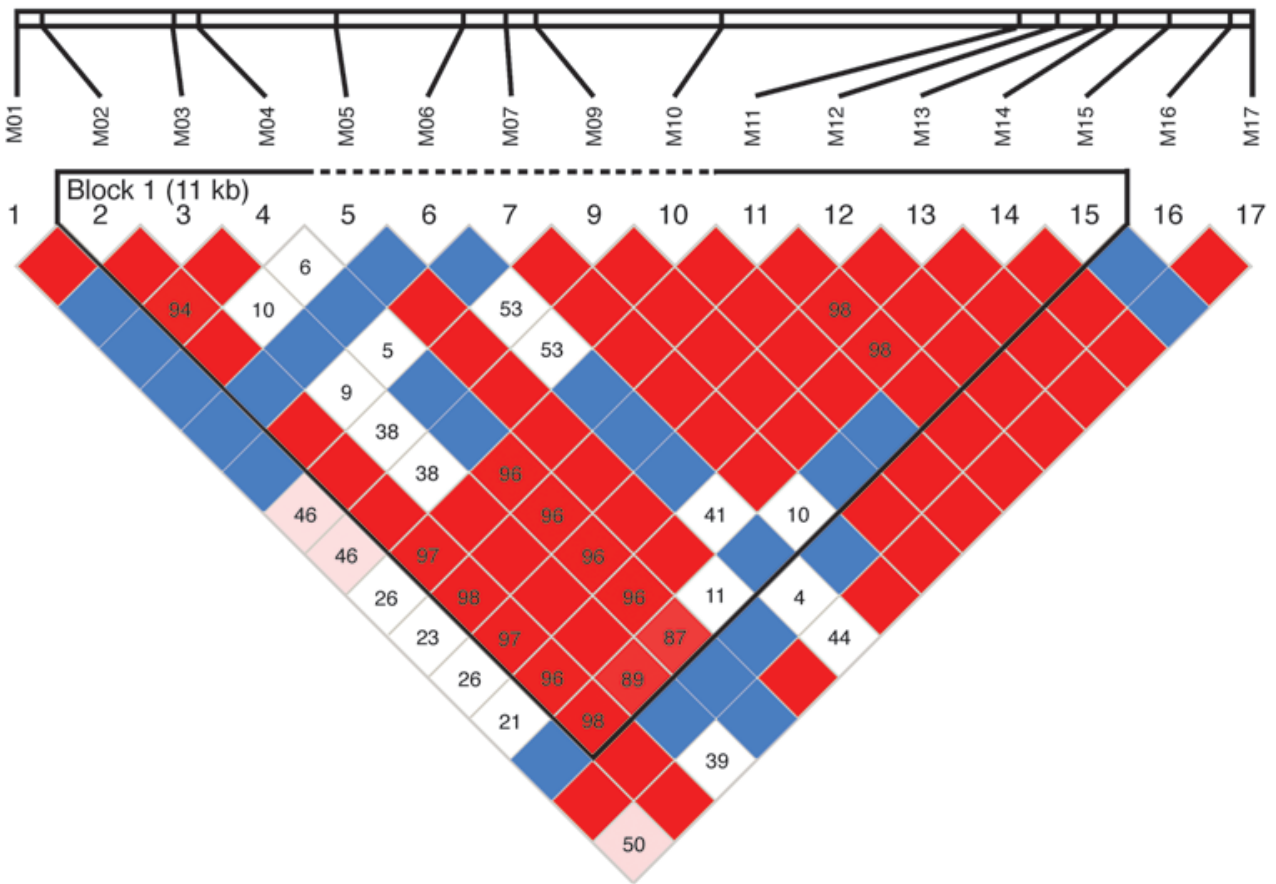

Figure 1

Genetic variation in the PPP1R1B region, showing the genotyped SNPs and their LD. Top row shows relative positions of the SNPs (see Table 1 for numbering). Below is a color-coded display of LD: strong LD (D'), red; weak LD, pink; weaker LD, white; not calculated, blue boxes. The haplotype block defined by the method of Gabrieli et al. that was used for association with biological and clinical phenotypes is marked by a black outline (output of the program Haploview). See Tables 1 and 2 and DNA collection and genotyping in Methods for details.

notype analyses (see below) and were in strong LD. The majority (approximately 75\%) of subjects carried a very frequent haplotype (CGCACTC); 1 other haplotype (GATGTCA) had sample frequencies around $15 \%$, and 3 other haplotypes were rare (Table 2 ).

Cognitive phenotypes. In the CBDB/NIMH family dataset, to search for evidence of functional relevance of the identified genetic variation in the gene, we used Family-Based Association Testing (FBAT) software version 1.7.2 to test for genetic association with cognitive performance in a sample of probands and their primarily unaffected siblings from our ongoing family-based study. The quantitative cognitive phenotypes were derived from a standard cognitive test battery for which heritability and association with schizophrenia (30) have been previously established. This analysis showed significant genotype effects on a range of phenotypes (Table 3 ), including general intelligence, measured by IQ and Wide Range Achievement Test (WRAT) reading; working memory, as assessed by the N-back test (1- to 3-back); Wisconsin Card Sorting Test (categories completed and perseverative errors t score) and letter fluency tests; and also sequencing, response alternation, and attention, as measured by the Gordon Continuous Performance Test D', trails B and trails A. All of these tests have been related to function of cortical striatal loops (18). In contrast, no association was found with tests of episodic memory, the California Verbal Learning Test, and Wechsler Memory Scale-Revised logical memory 1 and 2, tests that are traditionally related to function of temporal-diencephalic circuitry. No other tests of association with cognitive phenotypes were performed in this study. Most significant associations were found with M04, with better performance associated with the $\mathrm{G}$ allele. The same domains were associated with the 7-SNP haplotype, as expected, since it consisted of SNPs selected for cognitive association (Table 3).
Impact of genetic variation on gene expression. To investigate the impact of the observed genetic variation on functional gene regulation affecting expression, we studied the effect of the PPP $1 R 1 B$ haplotype on DARPP-32 mRNA abundance in an independent sample of postmortem human prefrontal cortices from 38 white subjects (22 controls and 16 subjects with schizophrenia). Several probes were used to identify known mRNA isoforms using quantitative PCR. Probe 14 identified all transcripts, probe 10 identified all transcripts except a truncated form previously described as t-DARPP (AY070271) (31), and probe 9667 identified all transcripts except for a splice variant (AK123112) missing exons 3 and 4. Probe 16 specifically identified t-DARPP, and probe 5183 identified the splice variant AK123112 (Figure 2A). In an analysis that controlled for the potentially confounding factors of sex, smoking, diagnostic status, postmortem interval, age, and RNA quality, the PPP1R1B haplotype identified from the cognitive associations had a significant impact, or trended

Table 2

Frequencies of 7-SNP haplotype in CBDB/NIMH samples

$\begin{array}{cccccccc}\text { M03 } & \text { M04 } & \text { M11 } & \text { M12 } & \text { M13 } & \text { M14 } & \text { M15 } & \text { Frequency } \\ \text { C } & \text { G } & \text { C } & \text { A } & \text { C } & \text { T } & \text { C } & 76.0 \% \\ \text { G } & \text { A } & \text { T } & \text { G } & \text { T } & \text { C } & \text { A } & 14.1 \% \\ \text { C } & \text { G } & \text { T } & \text { G } & \text { T } & \text { C } & \text { C } & 3.5 \% \\ \text { C } & \text { G } & \text { T } & \text { G } & \text { T } & \text { C } & \text { A } & 2.1 \% \\ \text { C } & \text { A } & \text { C } & \text { A } & \text { C } & \text { T } & \text { C } & 1.6 \%\end{array}$

Frequencies represent expectation maximization estimates from the FBAT program. See Figure 1 for map of gene and Table 1 for individual SNP information. Family-based association sample (257 families of white ancestry, CBDB/NIMH sample). 


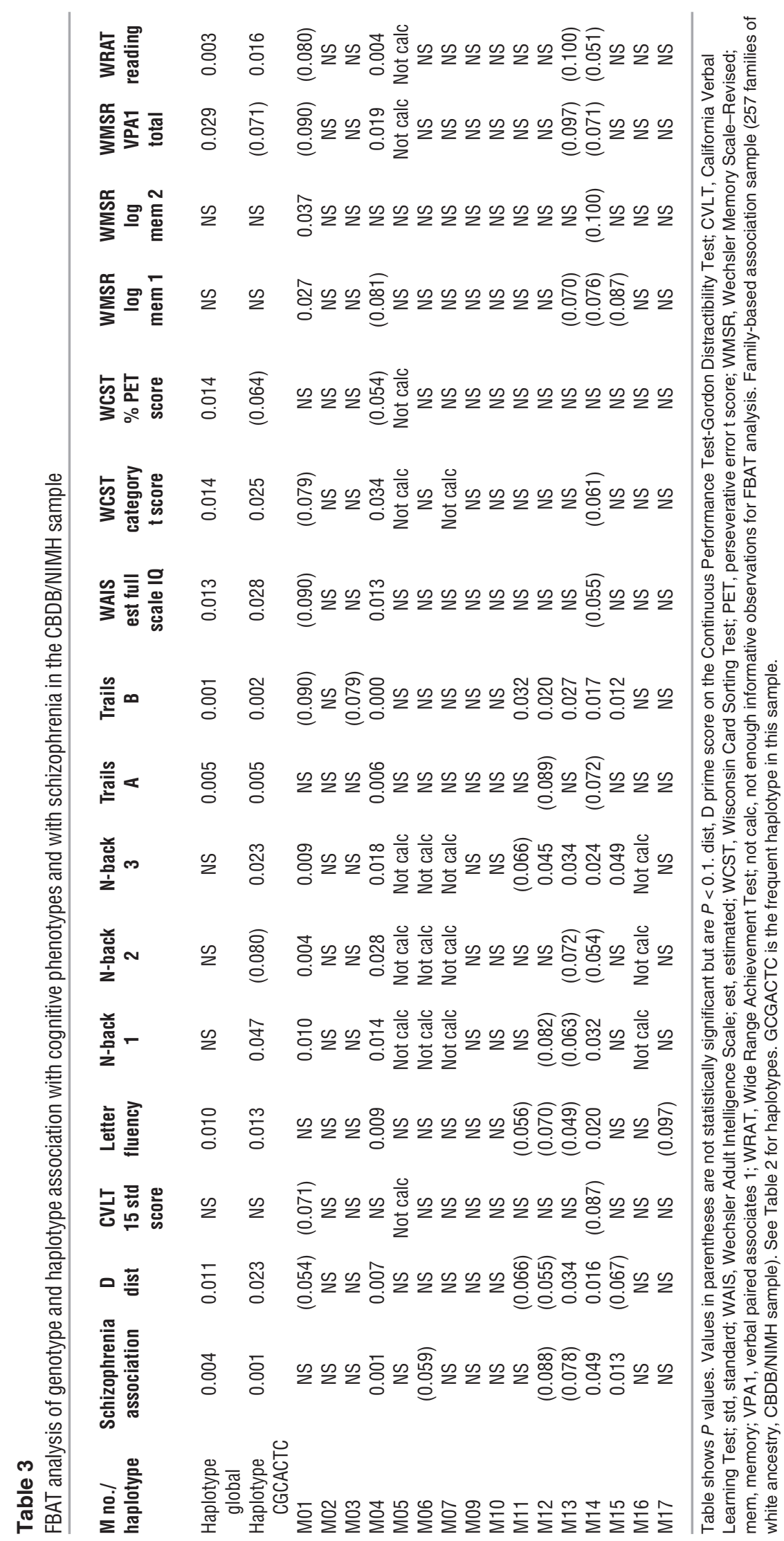

toward significance, on all probes assaying the expression of the full-length mRNA (type 14, $P<0.006$; type $10, P=0.09$; type 9967, $P<0.003)$. Expression was highest for homozygotes of the frequent (CGCACTC) haplotype, lowest for carriers of the less frequent (GATGTCA) haplotype, and intermediate for 2 subjects heterozygous for CGCACTC in combination with a rare haplotype (Figure $2 \mathrm{~B}$ ). There was no significant effect of diagnostic status, in agreement with recent observations (20). Probes specific for t-DARPP $(P=0.24)$ and the splice variant $5183(P=0.94)$ showed no significant association with haplotype. As a control, we ran the same analysis with the Val108/158 Met genotype in COMT. This had no significant effect on expression levels of PPP1R1B. These data implicate a genetic mechanism for cognitive associations at the level of transcriptional regulation, splicing, or mRNA stability.

Neuroimaging sample. The observed associations of genetic variation with cognitive tests related to frontostriatal function suggest a functional impact on this neural circuit. To investigate such systems-level correlates, we studied structure and function of the neostriatum and its interactions with prefrontal cortex (Figure 3 ) in an independent and large sample of healthy white volunteer subjects (96-142 subjects) not included in the cognitive test sample. Many of these subjects participated in both structural and functional neuroimaging; details of the demographics and subject overlap appear in Supplemental Table 1 (supplemental material available online with this article; doi:10.1172/JCI30413DS1).

Brain structure. First, we used voxel-based morphometry (VBM) in 96 individuals to canvas the brain for regional volume changes related to the haplotype derived from the cognitive associations and showing association with mRNA expression. This revealed a bilateral relative decrease in neostriatal volume (maximum in dorsal putamen) (Supplemental Table 2 and Figure 3A) for the frequent (CGCACTC) haplotype compared with all other haplotypes. In these subjects we then investigated "structural connectivity" of the bilateral striatum, a measure of covariation of structural volume data that we previously found related to cortical-subcortical connectivity influenced by genetic variation (32). We observed a pronounced increase in this measure of putative structural connectivity between striatum and lateral pre- 

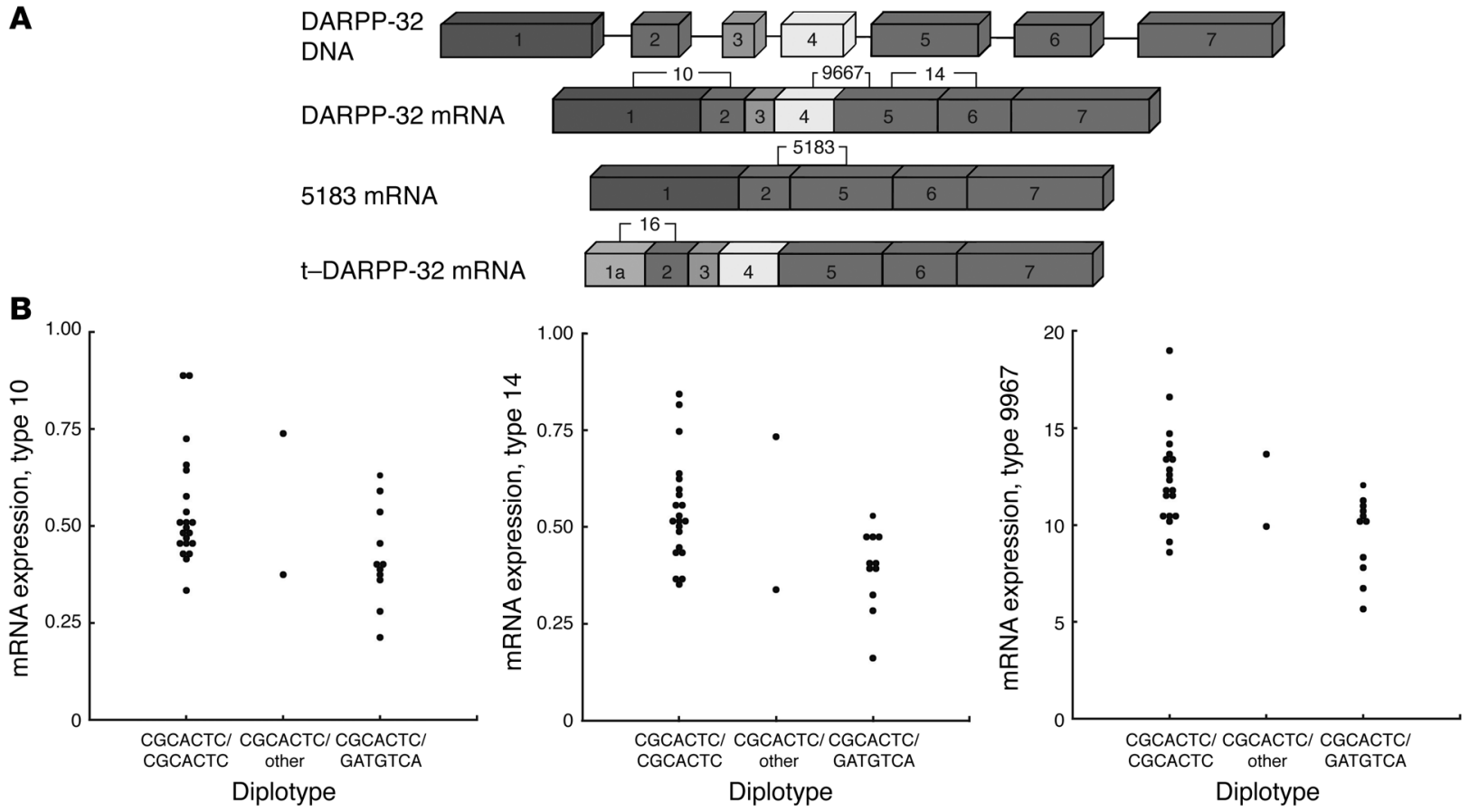

Figure 2

Effect of PPP1R1B haplotype on mRNA expression in postmortem human brain. (A) Exon position of primers used for quantitative PCR for isoform amplification, showing which isoform was amplified by which probe. (B) Effect of PPP1R1B haplotypes on mRNA expression for the common isoform. The PPP1R1B haplotype has an impact on expression of all probes assaying the expression of the full-length mRNA (type 14, $P<0.006$; type 10, $P=0.09$; type 9967, $P<0.003$ ). Expression was highest for homozygotes of the frequent (CGCACTC) haplotype (21 subjects), lowest for carriers of the less frequent (GATGTCA) haplotype (11 subjects), and intermediate for 2 subjects heterozygous for CGCACTC in combination with a rare haplotype. $\mathbf{B}$ is the ratio of DARPP expression to the geometric mean of expression of 3 housekeeping genes. See Gene expression in Methods for details.

frontal cortex, especially DLPFC, in carriers of the frequent haplotype (Supplemental Table 2 and Figure 3B).

Brain function. To probe functional consequences of this effect of genetic variation on brain structure and mRNA expression, we analyzed 2 large archival functional MRI (fMRI) datasets. While neither of these tasks was specifically designed to probe striatal function, both of them show differential activation in neostriatum in the context of prefrontal cortex involvement, as predicted by the frontostriatal loop concept (13). Performance during these tasks did not correlate with PPP1R1B CGCACTC haplotype (Supplemental Table 1). First, during a well-established working memory paradigm that robustly activates DLPFC, the N-back task (33), carriers of the frequent (CGCACTC) haplotype showed significantly less reactivity in the bilateral putamen (Supplemental Table 3 and Figure 3C), in regional agreement with the structural findings. Functional connectivity (a measure of correlation of brain activity over time) of the bilateral striatum showed a result similar to that of structural connectivity of the same region: connectivity with prefrontal cortex was strongly increased in carriers of the frequent haplotype (Supplemental Table 3 and Figure 3D). Next, we analyzed an emotional facematching task (FMT) using threatening visual stimuli (32), which probes neural circuitry of the human emotional alerting response and engages ventrolateral prefrontal and lateral orbitofrontal cortices (34). Again, we observed a significant effect of haplotype on striatal activation (caudate and putamen); as in the other fMRI task, carriers of the frequent haplotype showed decreased reactivity of the putamen (Supplemental Table 4 and Figure 3E). The analy- sis of the effects of haplotype on functional connectivity revealed a region in the left DLPFC that had increased connectivity for carriers of this haplotype (Supplemental Table 4 and Figure 3F). While it is expected that anatomical wiring and functional reactivity of the same brain circuits should bear some relationship to each other, since the structural and fMRI protocols and analyses performed here are independent acquisitions both technically and temporally and spatial normalization procedures ensure that the fMRI analyses are not confounded by the structural measures, these results provide convergent evidence for genetic impact on structure and function of frontostriatal circuitry. To exclude potential confounds by genotype effects on performance, we analyzed the effect of PPP1R1B haplotype on correct responses (in percentages) and reaction time (in seconds) for the 2 tasks. No significant effects were observed (FMT, percentage correct, $P=0.24$, reaction time, $P=0.27$; N-back, percentage correct, $P=0.47$, reaction time, $P=0.77$ ).

COMT control analysis in neuroimaging sample. As a genetic control, we also analyzed regional volume and functional activation in the sample of healthy white volunteers as a function of the COMT Val108/158 Met genotype, which was known for a large majority of participants (see Supplemental Table 5 for details of demographics). In contrast to the pronounced effects of PPP1R1B haplotype on our striatal imaging variables, neither striatal volume nor activation was significantly affected by genetic variation in COMT.

Family-based association with schizophrenia. We performed a familybased association study for schizophrenia in 257 white families of self-declared European ancestry from the CBDB/NIMH sample. 
A

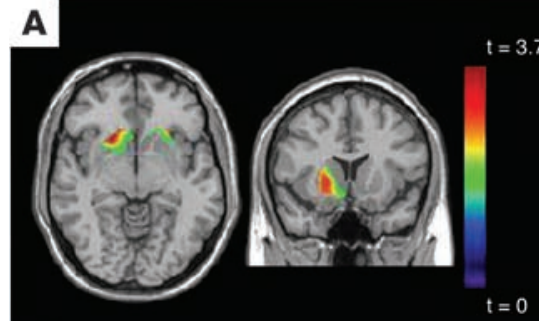

B

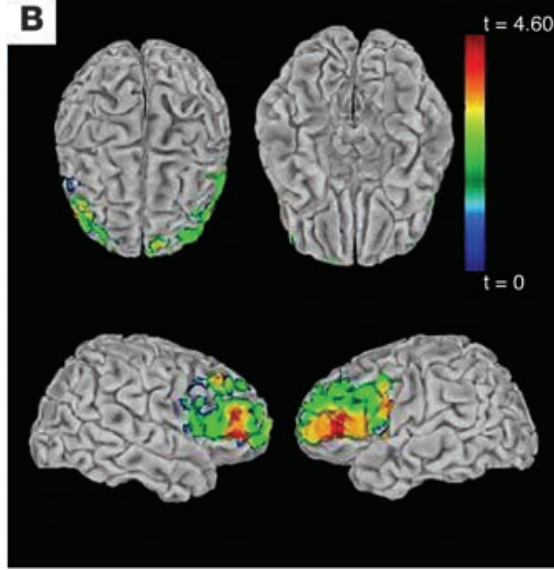

c

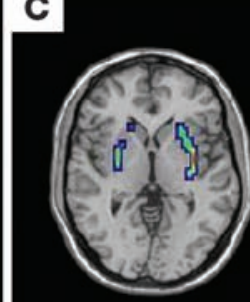

D

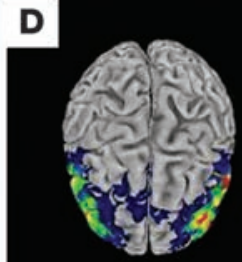

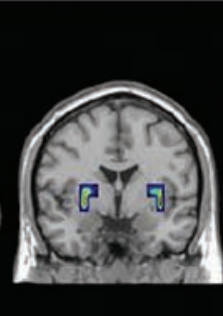

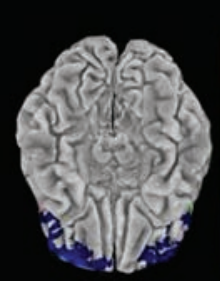

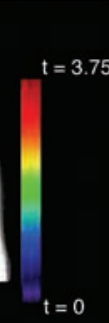

E
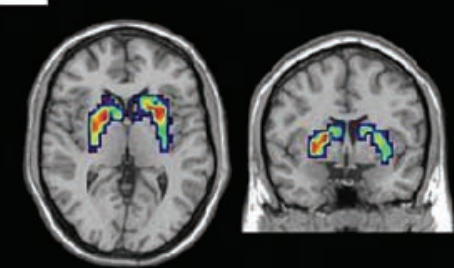
$t=3.75$

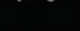

$F$
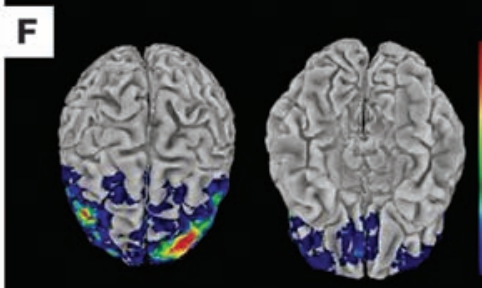

$=4.60$
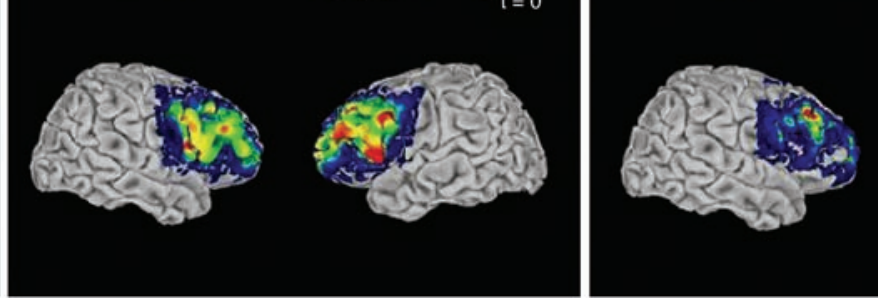

Figure 3

Neuroimaging analyses (structure and function) of common haplotype in PPP1R1B in control sample of white subjects. Top row shows haplotype effects on volume $(\mathbf{A})$ or activation $(\mathbf{C}$ and $\mathbf{E})$ in striatum; bottom row shows haplotype effects on structural (B) and functional (D and $\mathbf{F})$ connectivity of striatum with prefrontal cortex. Structural MRI analyses (voxel-based morphometry): (A) significantly reduced volume in striatum $(P<0.05)$ for carriers of the frequent $($ CGCACTC) haplotype; $(B)$ greater structural connectivity between prefrontal cortex and striatum for homozygotes for the frequent (CGCACTC) haplotype. fMRI, N-back task: $(\mathbf{C})$ significantly reduced reactivity in putamen $(P<0.05)$ for carriers of the frequent (CGCACTC) haplotype; (D) greater functional connectivity between prefrontal cortex and striatum for homozygotes for the frequent (CGCACTC) haplotype. FMRI, FMT: (E) significantly reduced reactivity in striatum $(P<0.05)$ for carriers of the frequent $(C G C A C T C)$ haplotype; (F) greater functional connectivity between prefrontal cortex and striatum for homozygotes for the frequent (CGCACTC) haplotype. t color scales depict statistical significance level (t statistical value). See Supplemental Tables 2-4 for detailed statistical information and Neuroimaging in Methods for methodological details.

We found evidence for an association between allelic variation in PPP1R1B and schizophrenia for the common haplotype that showed association in our various analyses of normal subjects (global, $P=0.004)$. The frequent haplotype CGCACTC, containing the M04 G allele, was positively associated with schizophrenia $(P=0.001)$, and the rare haplotype CACACTC, containing the M04 A allele, was negatively $(P=0.0003)$ associated with schizophrenia. For single SNPs, the strongest positive individual association ( $G$ allele, $P=0.001$ ) was found for SNP M04 (Table 3). Nominally significant association was also found for M14 (T allele, $P=0.049$ ) and M15 (C allele, $P=0.031$ ), which are in tight LD with M04 (Figure 3 ) and may therefore not represent an independent signal. There was also weak evidence for an association with SNP M06 (G allele, $P=0.059$ ).

We were unable to replicate association results using the white families from the NIMH Human Genetics Initiative dataset (haplotype CGCACTC frequency: 0.76, $P=0.73$, NS) (only 15 families had informative data).

\section{Discussion}

We used a translational genetics approach to investigate the relevance of DARPP-32 for aspects of human brain morphology and function. Resequencing of the gene revealed common single-nucleotide variants mostly within a single LD block, which we summarized for subsequent multilevel association analyses in a 7-SNP haplotype. These 7 SNPs were selected because they showed strong association to cognitive phenotypes implicated in cortical-striatal function and were then used as a haplotype to test other biologic associations in independent datasets. This strategy reduces the number of association tests in a complex phenotype dataset while at the same time increasing the likelihood that chromosomes with causative mutations/haplotypes are contrasted with other chromosomes. All uncovered variants were noncoding, in agreement with recent findings from a limited resequencing study in 50 Chinese probands (35). While it cannot be excluded that the effects observed in this study are due to several rare mutations that are coding, arose on the background of a common (ancestral) haplotype, and are in LD with the analyzed SNPs, we propose that the associations with cognition and brain structure and function observed in our dataset may reflect complex patterns of allelic heterogeneity that have an impact on expression. This is supported by the analysis of mRNA expression, which showed an impact of PPP1R1B genetic variation on the abundance of all identified full-length $P P P 1 R 1 B$ isoforms, compatible with the assumption of polymorphic functional regulatory elements. Expression was highest for subjects homozygous for the frequent (CGCACTC) haplotype and lowest for carriers of the GATGTCA haplotype. This provided a plausible mechanism for an impact of the genetic variation character- 
ized here on neuronal function, similar to observations in other psychiatric risk genes of noncoding marker haplotypes having effects on mRNA expression (36).

In an effort to bridge the gap from gene transcription to cognitive behavior, we studied the effects of the associated haplotype at the level of neural systems in another independent sample of healthy controls using neurobiological phenotypes plausibly connected to the cognitive phenotypes and to variation in gene expression. As reviewed, DARPP-32 is unambiguously linked to neostriatal function (11), which is, via interaction with prefrontal cortex, prominently implicated in a variety of cognitive domains (13). In agreement with the neurochemical anatomy of DARPP expression, we observed a substantial impact of genetic variation on neostriatal volume in structural neuroimaging. Volume was relatively reduced in dorsal putamen in carriers of the frequent haplotype. The dorsal putamen participates in interactions with the prefrontal cortex (13); in primates, this has been labeled the associative loop and includes the DLPFC, much of the caudate nucleus, and the precommisural putamen as well as the ventral anterior thalamus (15). Perturbing this circuit at any point may affect its function, and indeed, there are studies in primates and rats showing that dysfunction of the striatum produces behavioral deficits similar to those found in dysfunction of the prefrontal cortex (37). A genetic effect on striatal-prefrontal interaction was confirmed by an analysis of structural connectivity of the striatum, which showed a strong increase in its correlation with prefrontal cortex in the frequent haplotype. While it is important to bear in mind that this structural connectivity reflects covariation of regional volumes across subjects and does not directly quantify anatomical connections in white matter, we (32) and others (38) have previously observed patterns of structural connectivity that agree with known anatomical connectivity and are sensitive to genetic variation (32), and studies of conditions with known impact on anatomical connectivity have shown that structural covariance characterizes these processes in aging (39-41) and in schizophrenia (42). Furthermore, in the human optic system, it has been demonstrated directly that anatomically connected structures (i.e., optic tract, lateral geniculate nucleus, and primary visual cortex) covary in volume across individuals (43).

The structural findings were confirmed and extended by the results in functional imaging, where functional connectivity between striatum and prefrontal cortex was again shown to be increased in carriers of the frequent haplotype in both fMRI paradigms. Regarding striatal activation, the striatum showed less responsiveness to the environmental stimuli in carriers of the frequent haplotype in both tasks, suggesting that this finding indicates more efficient, intrastriatal processing. Indeed, the relatively smaller putaminal volume associated with this haplotype may also reflect more efficient circuitry. Since regional volume changes are taken into account when functional imaging data are spatially normalized and the main effect of task is a deactivation during working memory but an activation during face processing, these functional imaging results are not likely to be partial volume artifacts. Again, the localization of statistical maxima was in lateral putamen (also reaching significance in caudate for the face-processing task), in agreement with the structural findings and highlighting those regions receiving input from the DLPFC and premotor prefrontal cortex. It is important to note that while our tasks are cognitive and engage associative prefrontal-striatal circuitry broadly, our results highlight putamen as the component of the structural and functional circuit DARPP most has an impact on but not necessarily as the main striatal processing station related to task performance. Taken together, the neuroimaging data therefore show that the frequent haplotype is associated in healthy individuals with more efficient intrastriatal processing combined with an increase of prefrontal cortical input onto a smaller striatum. We note that the size reduction may contribute to striatal processing efficiency as it has been proposed that increasing overlap of functional projection fields by compression of pathways into successively smaller striatal structures is a key mechanism for the integration of information streams in the basal ganglia (15). Since our analyses of function concerned 2 tasks that were designed to assess prefrontal function during emotional and working memory processing, it would be useful to extend the present study by tasks that are specifically intended to activate striatal areas engaged in other cognitive functions, such as implicit memory or reward.

These systems-level findings provide a neural mechanism for the observed pronounced impact of genetic variation in $P P P 1 R 1 B$ on a wide range of cognitive domains during neuropsychological testing. The imaging findings confirm the behavioral importance of DARPP-32 effects on frontostriatal processing since working memory and response alternation and attention critically depend on both prefrontal and striatal function and are disturbed in diseases thought to have an impact on these structures, such as schizophrenia and Parkinson disease (18). Improved working memory and executive capacity may also underlie the association with general intelligence (44). The range of cognitive tests affected by variation in this gene likely reflects the critical importance of frontostriatal function for core aspects of cognition (18). As an important negative, 3 memory tasks depending on hippocampal but not striatal function, the California Verbal Learning Test and Wechsler Memory Scale-Revised logical memory 1 and 2 tests, were not affected by $P P P 1 R 1 B$ variation, suggesting a relatively specific impact on the frontostriatal circuit as well as a limited role of DARPP-32-dependent processing in neural systems for episodic memory in humans.

Our control analysis using the functional common Val108/158 Met genetic variation in COMT showed no effect on PPP1R1B mRNA expression, volume, or functional activation of the striatum, in good agreement with human (45) and animal knockout (26) data showing that this variant has an impact on dopamine turnover in prefrontal cortex but not striatum, where dopamine flux is mainly dependent on the dopamine transporter. These negative control findings, therefore, suggest that the biological associations with $P P P 1 R 1 B$ found in the present study are specifically related to the integrative function of DARPP-32 in dopaminoceptive striatal neurons and not to a general effect on dopaminergic neurotransmission elsewhere in this circuit.

Finally, we conducted a family-based association study to investigate an impact of the observed association with risk for schizophrenia. Since DARPP-32 has an impact on cortical plasticity, striatal function, dopaminergic neurotransmission, and response to psychotomimetics and neuroleptic treatment (3), it has long been viewed as an attractive candidate molecule for this disease. Its candidacy is further supported by our findings on frontostriatal processing in healthy controls since disinhibited dopaminergic neostriatal neurotransmission is associated with schizophrenia and has been linked to deficiencies in prefrontal function (27) and abnormal gating, proposed as a central mechanism in the disorder (16). Located at 17q21, PPP1R1B, the gene encoding DARPP-32, 
is also in or near a region implicated in risk for schizophrenia by a recent metaanalysis of whole genome linkage (46). Indeed, we found positive associations for the M04 G allele as well as for the frequent (CGCACTC) haplotype, the same alleles that had an impact on cognitive, expression, and imaging phenotypes.

This finding, however, must be regarded as preliminary since we did not have an adequately powered family-based replication sample, and it is therefore difficult to know whether this is a true association or a false positive. However, it is noteworthy that the alleles and haplotypes implicated in disease risk also predicted changes in controls mirroring observations in manifest schizophrenia. Striatal volumes in drug-naive, first-episode patients have been found to be decreased (47), an effect confounded later in the illness by neuroleptic treatment, which increases striatal volume (48). Imaging studies of patients with schizophrenia also show increased prefrontal-subcortical connectivity (49). This supports the tentative clinical association by showing that the directionality of the observed genetic effects agreed with the disease phenotype.

To the degree that this genetic association with schizophrenia can be confirmed, our data lead to the provocative observation that a frequent haplotype in PPP1R1B predicts increased frontostriatal interactions that appeared beneficial (as evidenced by relatively better performance on a wide range of cognitive tasks) yet contributed to risk for schizophrenia. This raises the question of whether a genetic advantage in normal subjects may translate into a disadvantage in the context of other functional impairments also associated with schizophrenia, such as abnormal function of the prefrontal cortex. To the degree that the striatum acts as an active gating station (50), increased information flow through this regulatory cognitive subsystem is predicted to contribute to increased flexibility, working memory capacity, and control capabilities, as observed in our normal subjects (51); on the other hand, in manifest schizophrenia, which is characterized by an inefficient, fractionated pattern of activation in prefrontal cortex (33), the same information-processing constellation could facilitate the persistence of disorganized cortical information and contribute to an escape of dysfunctional, unmodulated information from frontostriatal loops, leading to deficient cognition and inappropriate behavioral responses (16). Stated another way, the common DARPP-32 haplotype appears associated with optimized frontal-striatal function regardless of the specific information being processed through the system. The molecular processes by which genetic and environmental information regulate the development and modification of prefrontal-striatal circuitry involve synaptic plasticity, and DARPP-32 is a key molecule for shaping plasticity in striatal neurons receiving frontal afferents (52). Further work is necessary to confirm or refute this speculation.

In summary, we present convergent evidence in 3 independent datasets implicating DARPP-32 in a frontostriatal neural system for executive cognition and response selection in humans. We hope that this genetic identification of a molecular target at a critical nexus of dopaminergic neurotransmission and synaptic plasticity gives renewed impetus to the pursuit of therapeutic strategies aimed at postsynaptic signal integration in dopaminoceptive neurons that might benefit a diverse range of psychiatric disorders, notably addiction and schizophrenia.

\section{Methods}

Subjects. Five independent subject samples were used in this study. (a) For the initial sequencing effort to discover genetic variants, we resequenced DNA from 105 white and 44 African American patients with schizophre- nia, including the $9.7 \mathrm{~kb}$ of PPP1R1B composing the region of the gene spanned by its 7 exons and $2 \mathrm{~kb}$ upstream of the transcription start site. (b) For mRNA expression, postmortem brain samples were collected from 38 white subjects (22 controls and 16 schizophrenics). For genetic studies, probands with schizophrenia spectrum disorders, their unaffected siblings, and controls came from the Clinical Brain Disorders Branch Sibling Study, a study of neurobiological abnormalities related to genetic risk for schizophrenia (30). Only white people of European ancestry were studied to minimize heterogeneity and potential stratification artifacts. DNA was available for 257 white patients, 327 of their siblings, 397 parents of probands, and 243 controls. All subjects gave written informed consent and participated in the study according to the guidelines of the NIMH Institutional Review Board. Two independent samples from this genetic dataset were drawn for the present analyses. (c) Family data consisting of affected and unaffected offspring and parents were used for FBAT analyses (http://www.biostat. harvard.edu/ ${ }^{\mathrm{fbat}} / \mathrm{fbat} . \mathrm{htm}$ ) of disease and cognitive phenotypes. (d) An independent sample of healthy volunteer subjects was selected for neuroimaging after careful screening (30) to ensure they were free of any lifetime history of psychiatric or neurological illness, psychiatric treatment, or drug or alcohol abuse (Supplemental Table 1). The use in imaging of healthy subjects only is tailored to isolate gene effects independent of confounders related to illness, including chronicity, drug and alcohol use, smoking, medical treatment, and ongoing symptomatology. All available scans of subjects meeting these criteria (96-142 subjects; see Supplemental Table 1 for details) were used. (e) Finally, we also genotyped a selected sample of 67 white families from the NIMH Human Genetics Initiative (53) consisting of participants with the diagnosis of schizophrenia or schizoaffective disorder, depressed subtype, 1 or 2 siblings, and available parents. No other phenotypic measures were available for this sample.

DNA collection and genotyping. We used standard methods to extract DNA from white blood cells with the PUREGENE DNA purification kit (Gentra). We first resequenced material from 105 white and 44 African American patients with schizophrenia and identified 17 SNPs with minor allele frequencies greater than $3 \%$ in the $\mathrm{CBDB} / \mathrm{NIMH}$ family white dataset (Table 1). Genotype for only 1 SNP (M08) deviated from Hardy-Weinberg equilibrium in cases and controls; this SNP was discarded from further analyses. Genotype accuracy was assessed by regenotyping within a subsample, and reproducibility was routinely greater than $99 \%$. We eliminated probable genotyping errors within the family dataset through non-Mendelizing transmissions and haplotype inconsistency errors via MERLIN version 1.0.1 (http://www.sph.umich.edu/csg/abecasis/Merlin/download/). Genotypes based on assayed genotyping and on sequencing were identical. We measured LD between markers as indexed by the $\mathrm{D}^{\prime}$ and $\mathrm{R}^{2}$ statistics from case and control haplotypes with the program LDMAX within the Graphical Overview of Linkage Disequilibrium (GOLD) software package 2001 (54). To construct haplotypes for analyses of biological (functional imaging and gene expression) data, we used Haploview 3.2 software (http://www.broad. mit.edu/mpg/haploview/index.php) and assigned haplotypes to individuals using PHASE 2.1 (55). Haplotype frequencies are given in Figure 1.

Cognitive testing. To measure neurocognitive function, a cognitive test battery was administered to subjects from the $\mathrm{CBDB} / \mathrm{NIMH}$ family dataset (257 families). These cognitive tests, described previously (30), cover measures related to genetic risk for schizophrenia in the following domains: episodic memory (logical memory I and II from the Wechsler Memory Scale-Revised; and the California Verbal Learning Test), working memory (Wisconsin Card Sorting Test, categories and perseverative errors; and the $\mathrm{N}$-back test, 1-back and 2-back, given separately from the fMRI session), attention ( $D^{\prime}$ from the Gordon Continuous Performance Test), and letter fluency. To control for general intelligence, IQ (from the Wechsler Adult Intelligence Scale, revised edition) and reading comprehension (56) were 
also included. Selection of these cognitive phenotypes was based on evidence that they are related to genetic risk for schizophrenia (30). No other cognitive phenotypes were evaluated for this study. Single SNP and familybased haplotype analyses of these phenotypes were performed with FBAT version 1.7.2 using permutation testing for assessment of significance.

Gene expression. mRNA expression was studied using quantitative PCR. We employed 5 PPP1R1B probe/primer combinations on cDNA from postmortem brain samples collected from 38 white subjects $(22$ controls and 16 schizophrenics) after informed consent from the legal next of kin under NIMH protocol 90-M-0142. Gray matter tissue from the middle frontal gyrus was obtained from a coronal slab corresponding to the middle one-third immediately anterior to the genu of the corpus callosum. White matter was trimmed off. Tissue was pulverized and stored at $-80^{\circ} \mathrm{C}$. Total RNA was extracted from $300 \mathrm{mg}$ of tissue using the TRIzOL Reagent (Invitrogen). The yield of total RNA was determined by spectrophotometry, measuring absorbance at $260 \mathrm{~nm}$. RNA quality was assessed with high-resolution capillary electrophoresis (Agilent Technologies), and only samples showing clearly defined, sharp $18 \mathrm{~S}$ and $28 \mathrm{~S}$ ribosomal peaks, $28 \mathrm{~S} / 18 \mathrm{~S}$ ratios greater than 1.2 , and RNA integrity numbers greater than or equal to 4.0 were included. Total RNA $(4 \mu \mathrm{g})$ was used in $50 \mu \mathrm{l}$ of a reverse transcriptase reaction to synthesize cDNA by using the SuperScript First-Strand Synthesis System for RT-PCR (Invitrogen). Applied Biosystems Assays-on-Demand and custom-made primers/probes (for type 5183) were used to amplify specific transcripts based on the unique exon structure of each DARPP-32 isoform (17). Three probes were based on the common exon structure as found in full-length cDNA: exons 4 and 5 (type 9967; Hs00259967), 1 and 2 (type 10; Hs00938410_m1) and 5 and 6 (type 14; Hs00938414_m1); splice variant 5183, exons 2 and 5; and t-DARPP (type 16; Hs00938416_g1). The following Applied Biosystems Assays-on-Demand were used for measuring the levels of control genes for normalization of DARPP-32 expression data: porphobilinogen deaminase (PBGD; Hs006009297), $\beta_{2}$ microglobulin (B2M; Hs99999907), and $\beta$ glucuronidase (GUSB; Hs99999908). Expression levels of mRNAs were measured by real-time quantitative RT-PCR, using an ABI Prism 7900 Sequence Detection System with a 384-well format (Applied Biosystems). Each $10 \mu \mathrm{l}$ reaction contained $900 \mathrm{nM}$ of primer, $250 \mathrm{nM}$ of probe, and TaqMan Universal PCR Master Mix (Applied Biosystems) containing Hot GoldStar DNA Polymerase (Eurogentec), dNTPs with dUTP, uracil $\mathrm{N}$-glycosylase, passive reference, and 100-200 ng of cDNA template. PCR cycle parameters were $50^{\circ} \mathrm{C}$ for 2 minutes, $95^{\circ} \mathrm{C}$ for 10 minutes, 40 cycles of $95^{\circ} \mathrm{C}$ for 15 seconds, and $59^{\circ} \mathrm{C}$ or $60^{\circ} \mathrm{C}$ for 1 minute. PCR data were acquired from Sequence Detector Software (SDS version 2.0; Applied Biosystems) and quantified by a standard curve method using serial dilutions of pooled cDNA derived from RNA obtained from 10 to 12 control subjects. In each experiment, the R2 value of the curve was more than 0.99 , the slope was between -3.2 and -3.5 (amplification efficiency 96\%-101\%), and controls containing no-template cDNA resulted in no detectable signal. All samples were measured in a single plate for each target gene, and their threshold cycle $(\mathrm{Ct})$ values were in the linear range of the standard curve. All measurements were performed in triplicate. The data were normalized to the geometric mean of the control genes. All diplotypes containing at least 1 haplotype appearing in more than 1 subject were included in the analysis, leaving 34 subjects. Data were analyzed using the general linear model, with DARPP-32 diplotypes as predictors. Sex, smoking status, diagnostic status, postmortem interval, age, and RNA quality were also included in the regression model to account for variables unrelated to genotype that can potentially influence gene expression (57).

Neuroimaging - tasks. Both structural and functional neuroimaging were used to characterize neural function on the systems level. During fMRI scanning, subjects completed 2 tasks. The FMT is a simple perceptual task previously described to robustly engage the amygdala and hippocampal formation $(58,59)$. During 2 blocks of an emotion task, subjects viewed a trio of faces, selecting 1 of the 2 faces (bottom) that was identical to the target face (top). Per block, 6 images were presented sequentially for 5 seconds each, 3 of each sex and target affect (angry or afraid) derived from a standard set of pictures of facial affect. Emotion tasks alternated with 3 blocks of a sensorimotor control task in which faces were replaced with simple geometric shapes. The second task, the N-back (2-back version), probes working memory. It requires subjects to monitor a series of sequentially presented numerals randomly chosen from between 1 and 4 and, while continually receiving new numbers, press a corresponding button if the number presently on the screen is the same as that presented 2 items earlier. As a control task (0-back), subjects press a button corresponding to the number currently on the screen. This task has been used extensively (33) to study working memory in controls as well as patients with schizophrenia and their relatives and elicits robust DLPFC activation that is abnormal in patients. For both tasks, percentage of correct responses and reaction time were recorded. To investigate the effects of PPP1R1B haplotype on these performance parameters as potential confounds in neuroimaging, the general linear model was used with haplotypes assigned by PHASE as predictors and a contrast comparing the most frequent haplotype (CGCACTC) with the second most frequent haplotype (GATGTCA).

Neuroimaging: structural image processing. We acquired 3D structural MRI scans on a 1.5 Tesla GE scanner, using a T1-weighted spoiled grass sequence (repetition time[s]/excitation time[s]/number of excitations, $24 / 5 / 1$; flip angle, $45^{\circ}$; matrix size, $256 \times 256$ voxels; field of view, $24 \times 24 \mathrm{~cm})$ with 124 sagittal slices $(0.94 \times 0.94 \times 1.5 \mathrm{~mm}$ resolution $)$ preprocessed as previously described (60) followed by an optimized VBM protocol using customized templates $(61,62)$. Resulting gray matter images were smoothed with a $12-\mathrm{mm}$ Gaussian kernel prior to statistical analysis. Analysis was performed on a Linux workstation (Red Hat Enterprise) as implemented in MATLAB 6.52SP2 (MathWorks) and the general linear model (63) in SPM2 software (http://www.fil.ion.ucl.ac.uk/spm). The specification of parameters for the design matrix was described in detail elsewhere (60). In brief, effects of DARPP-32 genotype on gray matter volume were examined by using an analysis of covariance model adjusting for orthogonalized first- and second-order polynomials of age and sex. Haplotype effects were assessed as described below.

Neuroimaging: functional image processing. Blood oxygenation level-dependent (BOLD) fMRI was performed on a GE HealthCare Signa HDx 3.0T, using gradient echo-planar imaging (EPI) (24 axial slices, $4 \mathrm{~mm}$ thickness, $1 \mathrm{~mm}$ gap; TR/TE, $2,000 / 28 \mathrm{~ms}$; field of view, $24 \mathrm{~cm}$; matrix, $64 \times 64$ voxels). Images were processed as described previously $(58,59)$, using SPM99. In brief, images were realigned to the middle image of the scan run, spatially normalized into a standard stereotactic space (Montreal Neurological Institute template) using an affine and nonlinear $(4 \times 5 \times 4$ basis-functions) transformation, smoothed with an $8-\mathrm{mm}$ full width Gaussian filter at half maximum and ratio normalized to the whole-brain global mean. Statistical images for the contrast of the emotion task versus the sensorimotor control (for face matching) or 2-back minus 0-back (for N-back) were obtained for each subject. These maps were then analyzed for haplotype effects as described below.

Neuroimaging: functional connectivity analyses. Functional interactions with striatum were characterized using fMRI by measuring functional connectivity, as described previously $(60,64)$. This measurement examines the covariation across the brain with the activation in a region (volume) of interest (bilateral putamen). After mean and drift correction of the time series, median activity within this region of interest (ROI) was calculated (we prefer median as a robust estimator that coincides with the mean under the assumption of normality) for each scan. These values were then correlated across the brain 
with all voxel time series, resulting in a map which contained, in each voxel, the correlation coefficient of the time series in that voxel with that of the reference regions. These maps, 1 per subject, were then analyzed in a randomeffects model in SPM for haplotype effects as described below.

Neuroimaging: structural covariance analysis. Structural covariance examines structural coupling across individuals and employs a similar measure of coupling to functional connectivity, only this time not between functional data (fMRI time series), but between voxel-wise gray matter volume maps derived from VBM (see above). Voxels identified as significant in this approach have regional volume that is significantly positively or negatively correlated with the target area across subjects. We followed methods published previously (32). Summed volume in an anatomically defined ROI (bilateral putamen) in standardized space was computed by adding the local volume in all voxels comprising the ROI and used as a covariate of interest in a random-effects general linear model in SPM that also included nuisance covariates as described above for VBM.

Neuroimaging: second-level analysis of haplotype effects and statistical inference. After neuroimages were prepared for each subject by processing as described in the previous paragraphs, for all imaging methods, the analysis of haplotype effects was performed in a second-level random-effects model as previously described (24). The group level model used 1 contrast image per subject and regressed the estimated BOLD change on each subject's inferred haplotype frequencies. For statistical inference on the second level, we used the established methods of Gaussian random fields (GRF) theory (65). The significance threshold was set to $P<0.05$, corrected for multiple comparisons within hypothesis-driven ROI, defined by using the Wake Forest University School of Medicine PickAtlas (http://www.fmri.wfubmc. edu/download.htm). We used false discovery rate (FDR), as implemented in
SPM2, a frequentist method to control for type I error rate across the brain (66). For activation and volume, we investigated the striatum (caudate, putamen, and pallidum). For connectivity with striatum, we used a prefrontal cortex ROI encompassing Brodmann areas 9, 10, 32, 44, 45, and 46. Voxels significant at those thresholds are listed in Supplemental Tables 2-4. In Figures 1-3, all voxels affected by genotype within these regions are shown rendered on a representative control volume and surface. While all haplotypes were included in the statistical model, since comparisons with rarer haplotypes were underpowered in our imaging dataset, we compared only the most frequent haplotype (CGCACTC) and the second most frequent (GATGTCA) haplotype.

Association with schizophrenia. We tested for association with schizophrenia in 2 datasets: the CBDB/NIMH family dataset (257 families) and the NIMH Human Genetics Initiative family dataset (67 families). FBAT was used, with permutation testing for assessment of significance.

\section{Acknowledgments}

We would like to thank Courtnea Rainey for assistance in designing and drawing Figure 2A. This work was supported by the $\mathrm{NIMH} /$ Intramural Research Program.

Received for publication September 20, 2006, and accepted in revised form December 5, 2006.

Address correspondence to: Daniel R. Weinberger, NIH, Genes, Cognition and Psychosis Program, Room 4S-235, 10 Center Drive, Bethesda, Maryland 20892, USA. Phone: (301) 402-7564; Fax: (301) 480-7795; E-mail: weinberd@mail.nih.gov.
1. Walaas, S.I., Aswad, D.W., and Greengard, P. 1983. A dopamine- and cyclic AMP-regulated phosphoprotein enriched in dopamine-innervated brain regions. Nature. 301:69-71.

2. Hemmings, H.C., Jr., Greengard, P., Tung, H.Y., and Cohen, P. 1984. DARPP-32, a dopamine-regulated neuronal phosphoprotein, is a potent inhibitor of protein phosphatase-1. Nature. 310:503-505.

3. Svenningsson, P., et al. 2004. DARPP-32: an integrator of neurotransmission. Annu. Rev. Pharmacol. Toxicol. 44:269-296.

4. Goldman-Rakic, P.S. 1998. The cortical dopamine system: role in memory and cognition. Adv. Pharmacol. 42:707-711.

5. Schultz, W. 1998. Predictive reward signal of dopamine neurons. J. Neurophysiol. 80:1-27.

6. Weinberger, D.R. 1987. Implications of normal brain development for the pathogenesis of schizophrenia. Arch. Gen. Psychiatry. 44:660-669.

7. Grace, A.A. 2000. The tonic/phasic model of dopamine system regulation and its implications for understanding alcohol and psychostimulant craving. Addiction. 95(Suppl. 2):S119-S128.

8. Fiorillo, C.D., Tobler, P.N., and Schultz, W. 2003. Discrete coding of reward probability and uncertainty by dopamine neurons. Science. 299:1898-1902.

9. Svenningsson, P., et al. 2003. Diverse psychotomimetics act through a common signaling pathway. Science. 302:1412-1415.

10. Zachariou, V., et al. 2006. Phosphorylation of DARPP-32 at Threonine-34 is required for cocaine action. Neuropsychopharmacology. 31:555-562.

11. Ouimet, C.C., LaMantia, A.S., GoldmanRakic, P., Rakic, P., and Greengard, P. 1992. Immunocytochemical localization of DARPP-32, a dopamine and cyclic-AMP-regulated phosphoprotein, in the primate brain. J. Comp. Neurol. 323:209-218.

12. Ouimet, C.C., et al. 1984. DARPP-32, a dopamineand adenosine $3^{\prime}: 5^{\prime}$-monophosphate-regulated phosphoprotein enriched in dopamine-innervated brain regions. III. Immunocytochemical localization. J. Neurosci. 4:111-124.

13. Alexander, G.E., DeLong, M.R., and Strick, P.L. 1986. Parallel organization of functionally segregated circuits linking basal ganglia and cortex. Annu. Rev. Neurosci. 9:357-381.

14. Graybiel, A.M. 2005. The basal ganglia: learning new tricks and loving it. Curr. Opin. Neurobiol. 15:638-644.

15. Haber, S.N. 2003. The primate basal ganglia: parallel and integrative networks. J. Chem. Neuroanat. 26:317-330.

16. Swerdlow, N.R., Geyer,M.A., and Braff,D.L. 2001.Neural circuit regulation of prepulse inhibition of startle in the rat: current knowledge and future challenges. Psychopharmacology (Berl.). 156:194-215.

17. Dunnett, S.B., Meldrum, A., and Muir, J.L. 2005 Frontal-striatal disconnection disrupts cognitive performance of the frontal-type in the rat. Neuroscience. 135:1055-1065.

18. Pantelis, C., et al. 1997. Frontal-striatal cognitive deficits in patients with chronic schizophrenia. Brain. 120:1823-1843.

19. Albert, K.A., et al. 2002. Evidence for decreased DARPP-32 in the prefrontal cortex of patients with schizophrenia. Arch. Gen. Psychiatry. 59:705-712.

20. Baracskay, K.L., Haroutunian, V., and MeadorWoodruff, J.H. 2006. Dopamine receptor signaling molecules are altered in elderly schizophrenic cortex. Synapse. 60:271-279.

21. Meyer-Lindenberg, A., and Weinberger, D.R. 2006. Intermediate phenotypes and genetic mechanisms of psychiatric disorders. Nat. Rev. Neurosci. 7:818-827.

22. Harrison, P.J., and Weinberger, D.R. 2005. Schizophrenia genes, gene expression, and neuropathology: on the matter of their convergence. Mol. Psychiatry. 10:40-68.

23. Egan, M.F., et al. 2001. Effect of COMT Val108/158 Met genotype on frontal lobe function and risk for schizophrenia. Proc. Natl. Acad. Sci. U. S. A. 98:6917-6922.
24. Meyer-Lindenberg, A., et al. 2006. Impact of complex genetic variation in COMT on human brain function. Mol. Psychiatry. 11:867-877.

25. Tunbridge, E.M., Bannerman, D.M., Sharp, T., and Harrison, P.J. 2004. Catechol-o-methyltransferase inhibition improves set-shifting performance and elevates stimulated dopamine release in the rat prefrontal cortex. J. Neurosci. 24:5331-5335.

26. Gogos, J.A., et al. 1998. Catechol-O-methyltransferase-deficient mice exhibit sexually dimorphic changes in catecholamine levels and behavior. Proc. Natl. Acad. Sci. U. S. A. 95:9991-9996.

27. Meyer-Lindenberg, A., et al. 2002. Reduced prefrontal activity predicts exaggerated striatal dopaminergic function in schizophrenia. Nat. Neurosci. 5:267-271.

28. Kellendonk, C., et al. 2006. Transient and selective overexpression of dopamine D2 receptors in the striatum causes persistent abnormalities in prefrontal cortex functioning. Neuron. 49:603-615.

29. Gabriel, S.B., et al. 2002. The structure of haplotype blocks in the human genome. Science. 296:2225-2229.

30. Egan, M.F., et al. 2001. Relative risk for cognitive impairments in siblings of patients with schizophrenia. Biol. Psychiatry. 50:98-107.

31. El-Rifai, W., et al. 2002. Gastric cancers overexpress DARPP-32 and a novel isoform, t-DARPP. Cancer Res. 62:4061-4064.

32. Pezawas, L., et al. 2005. 5-HTTLPR polymorphism impacts human cingulate-amygdala interactions: a genetic susceptibility mechanism for depression. Nat. Neurosci. 8:828-834.

33. Callicott, J.H., et al. 2000. Physiological dysfunction of the dorsolateral prefrontal cortex in schizophrenia revisited. Cereb. Cortex. 10:1078-1092.

34. Meyer-Lindenberg, A., et al. 2005. Neural correlates of genetically abnormal social cognition in Williams syndrome. Nat. Neurosci. 8:991-993.

35. Li, C.H., Liao, H.M., Hung, T.W., and Chen, C.H. 2006. Mutation analysis of DARPP-32 as a candi- 
date gene for schizophrenia. Schizophr. Res. 87:1-5. 36. Law, A.J., et al. 2006. Neuregulin 1 transcripts are differentially expressed in schizophrenia and regulated by $5^{\prime}$ SNPs associated with the disease. Proc. Natl. Acad. Sci. U. S. A. 103:6747-6752.

37. Partiot, A., et al. 1996. Delayed response tasks in basal ganglia lesions in man: further evidence for a striato-frontal cooperation in behavioral adaptation. Neuropsychologia. 34:709-721.

38. Mechelli, A., Friston, K.J., Frackowiak, R.S., and Price, C.J. 2005. Structural covariance in the human cortex. J. Neurosci. 25:8303-8310.

39. Raz, N., et al. 2005. Regional brain changes in aging healthy adults: general trends, individual differences and modifiers. Cereb. Cortex. 15:1676-1689.

40. Raz, N., et al. 2004. Aging, sexual dimorphism, and hemispheric asymmetry of the cerebral cortex: replicability of regional differences in volume. Neurobiol. Aging. 25:377-396.

41. Raz, N., et al. 1997. Selective aging of the human cerebral cortex observed in vivo: differential vulnerability of the prefrontal gray matter. Cereb. Cortex. 7:268-282.

42. Woodruff, P.W., et al. 1997. Structural brain abnormalities in male schizophrenics reflect fronto-temporal dissociation. Psychol. Med. 27:1257-1266.

43. Andrews, T.J., Halpern, S.D., and Purves, D. 1997. Correlated size variations in human visual cortex lateral geniculate nucleus, and optic tract. J. Neurosci. 17:2859-2868

44. Gray, J.R., Chabris, C.F., and Braver, T.S. 2003. Neural mechanisms of general fluid intelligence. Nat. Neurosci. 6:316-322.

45. Lynch, D.R., et al. 2003. Lack of effect of polymorphisms in dopamine metabolism related genes on imaging of TRODAT-1 in striatum of asymptomatic volunteers and patients with Parkinson's disease. Mov. Disord. 18:804-812.
46. Lewis, C.M., et al. 2003. Genome scan meta-analysis of schizophrenia and bipolar disorder, part II: Schizophrenia. Am. J. Hum. Genet. 73:34-48.

47. Keshavan, M.S., Rosenberg, D., Sweeney, J.A., and Pettegrew, J.W. 1998. Decreased caudate volume in neuroleptic-naive psychotic patients. Am.J. Psychiatry. 155:774-778.

48. Chakos, M.H., et al. 1994. Increase in caudate nucle volumes of first-episode schizophrenic patients taking antipsychotic drugs. Am. J. Psychiatry. 151:1430-1436.

49. Meyer-Lindenberg, A.S., et al. 2005. Regionally specific disturbance of dorsolateral prefrontalhippocampal functional connectivity in schizophrenia. Arch. Gen. Psychiatry. 62:379-386.

50. Schneider, J.S. 1984. Basal ganglia role in behavior: importance of sensory gating and its relevance to psychiatry. Biol. Psychiatry. 19:1693-1710.

51. Miller, E.K., and Cohen, J.D. 2001. An integrative theory of prefrontal cortex function. Annu. Rev. Neurosci. 24:167-202.

52. Valjent, E., et al. 2005. Regulation of a protein phosphatase cascade allows convergent dopamine and glutamate signals to activate ERK in the striatum. Proc. Natl. Acad. Sci. U. S. A. 102:491-496.

53. Cloninger, C.R., et al. 1998. Genome-wide search for schizophrenia susceptibility loci: the NIMH Genetics Initiative and Millennium Consortium. Am. J. Med. Genet. 81:275-281.

54. Abecasis, G.R., and Cookson, W.O. 2000. GOLD - graphical overview of linkage disequilibrium. Bioinformatics. 16:182-183.

55. Stephens, M., Smith, N.J., and Donnelly, P. 2001. A new statistical method for haplotype reconstruction from population from population data. Am.J. Hum. Genet. 68:978-989.

56. Jastak, S., Wilkinson, G.S., and Jastak, J. 1984. Wide range achievement test - revised. Jastak Associates.
Wilmington, Delaware, USA.

57. Lipska, B.K., et al. 2006. Critical factors in gene expression in postmortem human brain: focus on studies in schizophrenia. Biol. Psychiatry. 60:650-658.

58. Hariri, A.R., Tessitore, A., Mattay, V.S., Fera, F., and Weinberger, D.R. 2002. The amygdala response to emotional stimuli: a comparison of faces and scenes. Neuroimage. 17:317-323.

59. Hariri, A.R., et al. 2005. A susceptibility gene for affective disorders and the response of the human amygdala. Arch. Gen. Psychiatry. 62:146-152.

60. Pezawas, L., et al. 2004. The brain-derived neurotrophic factor val66met polymorphism and variation in human cortical morphology. J. Neurosci. 24:10099-10102.

61. Augood, S.J., Westmore, K., and Emson, P.C. 1997. Phenotypic characterization of neurotensin messenger RNA-expressing cells in the neuroleptictreated rat striatum: a detailed cellular co-expression study. Neuroscience. 76:763-774.

62. Ashburner, J., and Friston, K.J. 2000. Voxelbased morphometry - the methods. Neuroimage. 11:805-821.

63. Friston, K.J., et al. 1995. Statistical parametric maps in functional imaging: a general linear approach. Hum. Brain Mapp. 2:189-210.

64. Meyer-Lindenberg, A., et al. 2001. Evidence for abnormal cortical functional connectivity during working memory in schizophrenia. Am. J. Psychiatry. 158:1809-1817

65. Worsley, K.J., et al. 1996. A unified statistical approach for determining significant signals in images of cerebral activation. Hum. Brain Mapp. 4:58-73.

66. Genovese, C.R., Lazar, N.A., and Nichols, T. 2002. Thresholding of statistical maps in functional neuroimaging using the false discovery rate. Neuroimage. 15:870-878. 